\title{
Author Index Volume 12 (2012/2013)
}

The issue number is given in front of the pagination

Agell, L., see de Muga, S. (1) 21-30

Akbulut, H., see Aktas, S.H. (3) 135-140

Akgun, N., see Aktas, S.H. (3) 135-140

Aktas, S.H., H. Akbulut, N. Akgun and F. Icli, Low dose chemotherapeutic drugs without overt cytotoxic effects decrease the secretion of VEGF by cultured human tumor cells: A tentative relationship between drug type and tumor cell type response (3) 135-140

Araki, Y., see Yoshitake, H. (3) 141-148

Araújo, A., see Catarino, R. (2) 65-72

Beck, A., see Joseph, S. (4,5) 177-184

Blasberg, J., see Joseph, S. (4,5) 177-184

Bocanek, O., see Faltejskova, P. (4,5) 199-204

Boonmars, T., see Rucksaken, R. (2) 81-95

Canis, M., A. Lechner, B. Mack, P. Zengel, R.P. Laubender, U. Koehler, V. Heissmeyer and O. Gires, CD133 is a predictor of poor survival in head and neck squamous cell carcinomas (2) 97105

Catarino, R., A. Coelho, A. Nogueira, A. Araújo, M. Gomes, C. Lopes and R. Medeiros, Cyclin D1 polymorphism in non-small cell lung cancer in a Portuguese population (2) 65-72

Chen, L., see Tang, X. (1) 11-19

Choi, K.Y., Y.S. Rho, K.H. Kwon, E.J. Chung, J.H. Kim, I.S. Park and D.J. Lee, ECRG1 and FGFR4 single nucleotide polymorphism as predictive factors for nodal metastasis in oral squamous cell carcinoma (3) 115-124

Chowdry, J., see Mackinder, M.A. (2) 49-64

Chung, E.J., see Choi, K.Y. (3) 115-124

Coelho, A., see Catarino, R. (2) 65-72

Corfe, B.M., see Mackinder, M.A. (2) 49-64

Dawood, S. and A.M. Gonzalez-Angulo, Biomarker discordance pre and post neoadjuvant chemotherapy in breast cancer (6) 241-250 de Muga, S., S. Hernández, M. Salido, M. Lorenzo, L. Agell, N. Juanpere, J.A. Lorente, S. Serrano and J. Lloreta, CXCR4 mRNA overexpression in high grade prostate tumors: Lack of association with TMPRSS2-ERG rearrangement (1) 21-30

Ding, X., see Huang, J. (3) 107-113

Ding, Y., see Shao, N. (1) 37-47

Donington, J., see Joseph, S. (4,5) 177-184

El Aaty Shawky, A., see El-Hafez, A.A. (4,5) 149-154

El-Hafez, A.A., A. El Aaty Shawky and B. Hasan, Cyclin D1 overexpression associates with favourable prognostic factors in invasive breast carcinoma $(4,5)$ 149-154

Endo, S., see Yoshitake, H. (3) 141-148

Evans, C.A., see Mackinder, M.A. (2) 49-64

Faltejskova, P., O. Bocanek, M. Sachlova, M. Svoboda, I. Kiss, R. Vyzula and O. Slaby, Circulating miR-17-3p, miR-29a, miR-92a and miR-135b in serum: Evidence against their usage as biomarkers in colorectal cancer $(4,5)$ 199-204

Fan, G., see Gao, Y. (1) 31-36

Fan, L., see Zou, Z.-J. (4,5) 169-176

Feng, N.H., see Shao, N. (1) 37-47

Fu, Q., see Quan, Y. (3) 125-133

Fujiwara, H., see Yoshitake, H. (3) 141-148

Gam, L.H., see Lim, S.R. (4,5) 185-198

Gao, C., see Huang, J. (3) 107-113

Gao, S., see Huang, J. (3) 107-113

Gao, Y., J. Wang and G. Fan, NPRL2 is an independent prognostic factor of osteosarcoma (1) 31-36

Gires, O., see Canis, M. (2) 97-105

Goel, R., see Kashyap, M.K. (1) 1-9

Goldberg, J.D., see Joseph, S. (4,5) 177-184

Gomes, M., see Catarino, R. (2) 65-72

Gong, Y., Significance of biomarker discordance in breast cancer from the pathologist's perspective (6) $207-218$ 
Gonzalez-Angulo, A.M., see Dawood, S. (6) 241-250

Gooi, B.-H., see Lim, S.R. (4,5) 185-198

Harrington, R., see Joseph, S. (4,5) 177-184

Harsha, H.C., see Kashyap, M.K. (1) 1-9

Hasan, B., see El-Hafez, A.A. (4,5) 149-154

Heissmeyer, V., see Canis, M. (2) 97-105

Hernández, S., see de Muga, S. (1) 21-30

Hiraku, Y., see Rucksaken, R. (2) 81-95

Hirsch, N., see Joseph, S. (4,5) 177-184

Hua, L.X., see Shao, N. (1) 37-47

Huang, J., C. Gao, X. Ding, S. Qu, L. Liu, W. Wu, L. Zhang, J. Zhao and S. Gao, MGB-based onestep multiplex real-time PCR method for rapid detection of HPV (3) 107-113

Icli, F., see Aktas, S.H. (3) 135-140

Ishikawa, H., see Yoshitake, H. (3) 141-148

Jiang, W.Y., see Shao, N. (1) 37-47

Joseph, S., R. Harrington, D. Walter, J.D. Goldberg, X. Li, A. Beck, T. Litton, N. Hirsch, J. Blasberg, M. Slomiany, W. Rom, H. Pass and J. Donington, Plasma osteopontin velocity differentiates lung cancers from controls in a CT screening population (4,5) 177-184

Juanpere, N., see de Muga, S. (1) 21-30

Kashyap, M.K., H.A. Pawar, S. Keerthikumar, J. Sharma, R. Goel, R. Mahmood, M.V. Kumar, K.V.V. Kumar, A. Pandey, R.V. Kumar, T.S.K. Prasad and H.C. Harsha, Evaluation of protein expression pattern of stanniocalcin 2, insulin-like growth factorbinding protein 7 , inhibin beta $\mathrm{A}$ and four and a half LIM domains 1 in esophageal squamous cell carcinoma (1) 1-9

Keerthikumar, S., see Kashyap, M.K. (1) 1-9

Khan, S., see Lower, E.E. (6) 219-230

Khoontawad, J., see Rucksaken, R. (2) 81-95

Kim, D.S., see Na, Y. (2) 73-79

Kim, J.H., see Choi, K.Y. (3) 115-124

Kiss, I., see Faltejskova, P. (4,5) 199-204

Koehler, U., see Canis, M. (2) 97-105

Kumar, K.V.V., see Kashyap, M.K. (1) 1-9

Kumar, M.V., see Kashyap, M.K. (1) 1-9

Kumar, R.V., see Kashyap, M.K. (1) 1-9

Kwon, K.H., see Choi, K.Y. (3) 115-124

Laubender, R.P., see Canis, M. (2) 97-105

Lechner, A., see Canis, M. (2) 97-105

Lee, D.J., see Choi, K.Y. (3) 115-124
Lee, S.M., see Na, Y. (2) 73-79

Li, F., see Tang, X. (1) 11-19

Li, H., see Tang, X. (1) 11-19

li, J.-Y., see Zou, Z.-J. (4,5) 169-176

Li, X., see Joseph, S. (4,5) 177-184

Lim, S.R., B.-H. Gooi and L.H. Gam, Identification of low abundance proteins in colorectal cancer tissues (4,5) 185-198

Litton, T., see Joseph, S. (4,5) 177-184

Liu, L., see Huang, J. (3) 107-113

Lloreta, J., see de Muga, S. (1) 21-30

Lopes, C., see Catarino, R. (2) 65-72

Lorente, J.A., see de Muga, S. (1) 21-30

Lorenzo, M., see de Muga, S. (1) 21-30

Lower, E.E. and S. Khan, Biomarker discordance: Why it occurs and why it is important (6) 219-230

Mack, B., see Canis, M. (2) 97-105

Mackinder, M.A., C.A. Evans, J. Chowdry, C.A. Staton and B.M. Corfe, Alteration in composition of keratin intermediate filaments in a model of breast cancer progression and the potential to reverse hallmarks of metastasis (2) 49-64

Mahmood, R., see Kashyap, M.K. (1) 1-9

Maruyama, M., see Yoshitake, H. (3) 141-148

Medeiros, R., see Catarino, R. (2) 65-72

Moussa, O., C. Purdie, S. Vinnicombe and A.M. Thompson, Biomarker discordance: Prospective and retrospective evidence that biopsy of recurrent disease is of clinical utility (6) 231-239

Na, Y., S.M. Lee, D.S. Kim and J.Y. Park, Promoter methylation of Wnt antagonist $D K K 1$ gene and prognostic value in Korean patients with nonsmall cell lung cancers (2) 73-79

Niikura, N. and N.T. Ueno, Change in HER2 status during breast tumor progression (6) 251-255

Nogueira, A., see Catarino, R. (2) 65-72

Nojima, M., see Yoshitake, H. (3) 141-148

Pairojkul, C., see Rucksaken, R. (2) 81-95

Pandey, A., see Kashyap, M.K. (1) 1-9

Park, I.S., see Choi, K.Y. (3) 115-124

Park, J.Y., see Na, Y. (2) 73-79

Pass, H., see Joseph, S. (4,5) 177-184

Pawar, H.A., see Kashyap, M.K. (1) 1-9

Pinlaor, P., see Rucksaken, R. (2) 81-95

Pinlaor, S., see Rucksaken, R. (2) 81-95

Prasad, T.S.K., see Kashyap, M.K. (1) 1-9

Purdie, C., see Moussa, O. (6) 231-239 
Qiao, D., see Shao, N. (1) 37-47

Qu, S., see Huang, J. (3) 107-113

Quan, Y., Y. Yan, X. Wang, Q. Fu, W. Wang, J. Wu, G. Yang, J. Ren and Y. Wang, Impact of cell dissociation on identification of breast cancer stem cells (3) 125-133

Ren, G., see Tang, X. (1) 11-19

Ren, J., see Quan, Y. (3) 125-133

Rho, Y.S., see Choi, K.Y. (3) 115-124

Rom, W., see Joseph, S. (4,5) 177-184

Roytrakul, S., see Rucksaken, R. (2) 81-95

Rucksaken, R., J. Khoontawad, S. Roytrakul, P. Pinlaor, Y. Hiraku, C. Wongkham, C. Pairojkul, T. Boonmars and S. Pinlaor, Proteomic analysis to identify plasma orosomucoid 2 and kinesin 18A as potential biomarkers of cholangiocarcinoma (2) $81-95$

Sachlova, M., see Faltejskova, P. (4,5) 199-204

Salido, M., see de Muga, S. (1) 21-30

Serrano, S., see de Muga, S. (1) 21-30

Shao, N., W.Y. Jiang, D. Qiao, S.G. Zhang, Y. Wu, X.X.Zhang, L.X. Hua, Y. Ding and N.H. Feng, An updated meta-analysis of XRCC4 polymorphisms and cancer risk based on 31 case-control studies (1) $37-47$

Sharma, J., see Kashyap, M.K. (1) 1-9

Slaby, O., see Faltejskova, P. (4,5) 199-204

Slomiany, M., see Joseph, S. (4,5) 177-184

Staton, C.A., see Mackinder, M.A. (2) 49-64

Suzuki, F., see Yoshitake, H. (3) 141-148

Svoboda, M., see Faltejskova, P. (4,5) 199-204

Takamori, K., see Yoshitake, H. (3) 141-148

Tang, X., X. Yin, T. Xiang, H. Li, F. Li, L. Chen and G. Ren, Protocadherin 10 is frequently downregulated by promoter methylation and functions as a tumor suppressor gene in non-small cell lung cancer (1) 11-19

Thompson, A.M., see Moussa, O. (6) 231-239

Ueno, N.T., see Niikura, N. (6) 251-255

Vinnicombe, S., see Moussa, O. (6) 231-239

Vyzula, R., see Faltejskova, P. (4,5) 199-204

Walter, D., see Joseph, S. (4,5) 177-184
Wang, J., see Gao, Y. (1) 31-36

Wang, L., see Zou, Z.-J. (4,5) 169-176

Wang, W., see Quan, Y. (3) 125-133

Wang, X., see Quan, Y. (3) 125-133

Wang, Y., see Quan, Y. (3) 125-133

Wongkham, C., see Rucksaken, R. (2) 81-95

Wu, J., see Quan, Y. (3) 125-133

Wu, W., see Huang, J. (3) 107-113

Wu, Y., see Shao, N. (1) 37-47

Xiang, T., see Tang, X. (1) 11-19

Xu, W., see Zou, Z.-J. (4,5) 169-176

Yan, Y., see Quan, Y. (3) 125-133

Yang, G., see Quan, Y. (3) 125-133

Yang, S., see Zou, Z.-J. (4,5) 169-176

Yin, X., see Tang, X. (1) 11-19

Yokoi, H., see Yoshitake, H. (3) 141-148

Yoshida, K., see Yoshitake, H. (3) 141-148

Yoshikawa, H., see Yoshitake, H. (3) 141-148

Yoshitake, H., H. Yokoi, H. Ishikawa, M. Maruyama, S. Endo, M. Nojima, K. Yoshida, H. Yoshikawa, F. Suzuki, K. Takamori, H. Fujiwara and Y. Araki, Overexpression of TEX101, a potential novel cancer marker, in head and neck squamous cell carcinoma (3) 141-148

Zeng, B., see Zhang, C. (4,5) 155-167

Zengel, P., see Canis, M. (2) 97-105

Zhang, C., Y. Zhao and B. Zeng, Enhanced chemosensitivity by simultaneously inhibiting cell cycle progression and promoting apoptosis of drugresistant osteosarcoma MG63/DXR cells by targeting Cyclin D1 and Bcl-2 (4,5) 155-167

Zhang, L., see Huang, J. (3) 107-113

Zhang, L.-N., see Zou, Z.-J. (4,5) 169-176

Zhang, R., see Zou, Z.-J. (4,5) 169-176

Zhang, S.G., see Shao, N. (1) 37-47

Zhang, X.X., see Shao, N. (1) 37-47

Zhao, J., see Huang, J. (3) 107-113

Zhao, Y., see Zhang, C. (4,5) 155-167

Zou, Z.-J., L. Fan, L. Wang, R. Zhang, L.-N. Zhang, S. Yang, J.-Y. li and W. Xu, Serum carbohydrate antigen 125 is not an independent prognostic factor in patients with chronic lymphocytic leukemia $(4,5)$ 169-176 